\title{
Que penser des études de cas pour la formation universitaire?
}

\section{Marianne Kugler}

\section{(2) OpenEdition}

1 Journals

Édition électronique

URL : http://journals.openedition.org/communicationorganisation/1891

DOI : 10.4000/communicationorganisation. 1891

ISSN : $1775-3546$

Éditeur

Presses universitaires de Bordeaux

\section{Édition imprimée}

Date de publication : 1 novembre 1996

ISSN : 1168-5549

\section{Référence électronique}

Marianne Kugler, "Que penser des études de cas pour la formation universitaire? », Communication et organisation [En ligne], 10 | 1996, mis en ligne le 26 mars 2012, consulté le 01 mai 2019. URL : http:// journals.openedition.org/communicationorganisation/1891; DOI : 10.4000/ communicationorganisation. 1891

Ce document a été généré automatiquement le 1 mai 2019.

(c) Presses universitaires de Bordeaux 


\title{
Que penser des études de cas pour la formation universitaire?
}

\author{
Marianne Kugler
}

1 Cet article est une réflexion sur le transfert des connaissances des praticiens aux étudiants. Après avoir passé plus de 15 ans dans le domaine des communications et des relations publiques, je passe en 1994 du monde des praticiens à celui des enseignants, des chercheurs, des analystes et des théoriciens. Deux mondes beaucoup plus éloignés que je ne le pensais. J'essaierai dans ce texte, avec le recul de seulement deux années d'enseignement, de préciser les choix méthodologiques qu'a susciter le cours : Études de cas en relations publiques.

\section{Les études de cas : problématique et fondement théorique}

2 Une étude de cas est définie comme " une stratégie descriptive basée sur un cas unique » (Gauthier 1993; p 564). Méthode qualitative, elle s'intéresse aux données descriptives comme le comportement des personnes ou des organisations, et empirique, elle se base essentiellement sur l'expérience des acteurs.

3 Les premières études de cas ont été faites par les médecins, pour partager leurs critères de diagnostics et leurs façons de traiter les malades. L'approche « clinique » qui a ensuite été adoptée en dehors du cercle des sciences de la santé fait référence au fait que le chercheur tient compte du traitement et de l'observation du patient et non d'une étude expérimentale ou de laboratoire. ${ }^{1}$

4 Les études de cas ont été adoptées par d'autres professions dont celles des communications. Mais elles ne sont pas seulement des outils de partage d'un savoir professionnel et, par dérive, des outils pédagogiques, elles sont aussi utilisées comme outils de recherche. Les anthropologues, les ethnologues et les sociologues, tous ceux qui ont été inspirés par la démarche de Max Weber, les ont utilisées². 
5 Les sciences naturelles font aussi largement appel au terrain dans leur démarche de recherche. Ce sont autant d'exemples d'études de cas. L'étude détaillée d'un écosystème ou d'une coupe géologique relève plus d'une stratégie descriptive d'un cas unique que d'une approche basée sur une théorie. Chaque coupe, chaque écosystème est étudié pour lui-même. Ensuite, il est analysé avec d'autres en tant qu'ensemble et ce regroupement, enrichi d'un apport théorique, permet parfois d'avoir une image globale ou régionale qui vient confirmer ou infirmer une théorie.

\section{Les études de cas comme outils de recherche en communication}

$6 \quad$ Les études de cas comme d'autres méthodes de recherche suivent les caprices des modes et, si on se fie à ce qu'en disent les manuels de méthodologie en communication, leur popularité a varié au cours des années et selon les auteurs. Hsia (1988) en fait une critique sévère: un manque de rigueur incompatible avec la méthode scientifique, une impossibilité ou une faible possibilité de conduire à des généralisations, une crainte que la subjectivité du chercheur influence ou biaise les informations et finalement le manque de moyens pour comprendre les conclusions opposées de différentes études de cas. Du côté positif, il reconnaît aux études de cas des qualités de discrétion, de non-intervention dans le déroulement des événements, de possibilité d'étudier toutes les facettes d'un cas et, ce qui n'est pas négligeable en ces périodes de manque de fonds de recherche, d'être relativement peu coûteuses. Il mentionne aussi que la multiplication des études de cas permet de voir se dessiner des typologies et, si ces études de cas se succèdent, s'échelonnent dans le temps, elles peuvent faire apparaître des tendances. Bowers, (1984) était plus enthousiaste et soulignait que le chercheur qui utilise cette méthode se rapproche de l'ethnologue et cherche à développer, en communication, ce qui a été développé par les sociologues : de la théorie ancrée dans la réalité (grounded theory)3.

7 À la même époque, des sociologues défendent avec ardeur les approches de type clinique en recherche (Berg 1985). Ceux-ci suggèrent que le chercheur qui utilise ces méthodes ait une relation au phénomène étudié et idéalement en ait une compréhension approfondie (Lowman, 1985). Comme la collecte des données ne se limite pas à des faits quantifiables qui peuvent être analysés statistiquement, elle est beaucoup plus large et particulièrement adaptée à l'appréhension de systèmes complexes. De plus le « clinicien » n'est pas lié à des concepts théoriques ou à des paradigmes prédéterminés ce qui lui donne un plus grande liberté d'observation et d'interprétation.

Dans les manuels plus récents de méthodologie des communications, les études de cas n'ont pas meilleure réputation. Soit, il n'en est tout simplement pas question, ce qui est le cas de la plupart des livres publiés dans le monde francophone (Laramée, Vallée, 1991; Massé, 1992), soit, elles sont considérées comme des méthodes de recherche extrêmement peu efficaces et, de plus, pouvant mener à des erreurs. À la limite, si le chercheur y tient vraiment, elles pourraient être utiles pour servir de point de départ à la mise au point d'hypothèses de recherche (Watt, 1995).

9 Ces réticences des auteurs de manuels ne semblent pas perturber outre mesure les chercheurs. Il suffit de regarder les sommaires des derniers numéros des revues en communication pour se convaincre que les études de cas sont largement utilisées et qu'il se publie aussi des monographies qui font date. 


\section{L'étude de cas : un outil de formation} place dans un programme d'enseignement universitaire de type professionnel. Dans le cadre du programme d'étude en communication publique à l'Université Laval, les étudiants qui choisissent le profil «relations publiques et publicité sociale " suivent un certain nombre de cours théoriques dans les premiers semestres puis des cours techniques pour terminer par un stage qui, malheureusement, à cause du trop grand nombre d'étudiants, n'est pas obligatoire.

11 La place du cours d'études de cas dans ce cursus est particulière et importante. Il s'agit, vers la fin du programme, d'un cours «synthèse » qui explique comment, dans la vraie vie, les spécialistes des différents domaines de la communication agencent et enchaînent analyses, réalisations et évaluations dans le cadre d'un cas précis. première où l'activité est centrée sur l'étudiant, consiste à donner à celui-ci une problématique à résoudre et une description détaillée de l'organisation et de la situation de départ. C'est ce modèle d'études de cas qui est utilisé dans les écoles d'administration type Harvard Business School. Cependant, elle n'est pas transportable dans un environnement où il y a beaucoup d'étudiants et un encadrement minimal. La deuxième façon d'utiliser les études de cas pour la formation est centrée sur l'acteur : celui qui a vécu le cas et qui, changeant de rôle, vient le décrire ${ }^{4}$ en cours à l'Université Laval. C'est cette deuxième façon qui s'est imposée d'elle-même étant donné le nombre d'étudiants et le manque d'encadrement adéquat pour les faire travailler en petits groupes.

\section{Quel cas?}

14 Le cours comprend une dizaine de «leçons » de trois heures qui forment un cours d'un semestre ${ }^{5}$. À chaque leçon, un conférencier vient «démonter " pour les étudiants les rouages d'une opération de communication : du début à la fin : mise en contexte, analyse de la problématique, etc. jusqu'à l'évaluation de l'efficacité de l'opération.

Le choix d'études-cas caractéristiques est fait d'abord de manière à rendre compte des différentes facettes des communications et des relations publiques. Il s'agit de trouver des exemples des grandes tendances de la pratique - utilisation des nouvelles technologies des communications-, de situations courantes, - communications internes, services à la clientèle, gestion de crise, organisation de levées de fonds. Mais aussi en tenant compte du type d'organisations : institutions gouvernementales, entreprises privées en situation de monopole ou non, organisations à but non lucratif etc.

Le choix est aussi largement influencé par l'actualité, ce qui permet de rattacher le cas à ce qui peut se lire dans la presse au moment où le cas sera présenté et de faire appel à la mémoire qu'en ont les étudiants - par exemple : le cas de la candidature de la Ville de Québec pour les Jeux Olympiques d'hiver de 2002, la restructuration des services de communication à Hydro-Québec, la perte du monopole de Bell dans la téléphonie

Communication et organisation, $10 \mid 2012$ 
interurbaine, les coupures dans les services de santé etc. Enfin, vu le grand nombre de cas possibles, les qualités pédagogiques de la personne qui viendra présenter le cas sont aussi déterminantes.

\section{Par qui ?}

Délibérément, le choix a été de faire de l'empirisme en demandant à un acteur et non à un analyste de présenter le cas. Directeur des communications, responsable de projet ou encore dirigeant de l'entreprise, l'acteur vient partager une expérience pratique ${ }^{6}$. Ce choix délibéré permet en premier lieu de rendre le cours plus vivant et, en deuxième lieu, plus proche de « la réalité » de ce que les étudiants auront à faire une fois sur le marché du travail. Il ne faut pas s'attendre en effet à ce qu'ils puissent pratiquer sur le terrain avec le détachement intellectuel de l'analyste. Ceci donne une coloration particulière au cours (Gingras, 1993). Si l'on se réfère à Goffman (1991) l'étude de cas relève dans ce cas-ci du cadre du théâtre ${ }^{7}$. La situation est même plus complexe puisqu'il y a un double cadre : celui de l'acteur dans son organisation et celui, temporaire, de l'acteur dans la salle de cours face aux étudiants.

Le cadre pédagogique peut venir perturber le cadre de l'acteur et réciproquement, surtout lors de la période de questions. Il arrive que l'acteur institutionnel se sentant interpellé par l'intervention d'un étudiant, remette en question lui-même les actions de son institution et les siennes. Par ailleurs, pour des raisons de respect d'allégeance ou même d'éthique, l'acteur ne pourra pas divulguer certaines informations qui auraient grandement éclairé pédagogiquement sa présentation aux étudiants.

L'expérience montre que si l'invitation est faite à plus d'un acteur de venir présenter le cas, en collaboration - par exemple le directeur des communication et son adjoint-, le résultat est moins bon à cause justement de cette multiplication des cadres et donc des sources de conflits possible entre eux.

\section{Comment ?}

20 Tous les cas sont présentés en utilisant, dans les grandes lignes, le même plan: description de l'organisation, état de la situation, problématique de communication, stratégie de la campagne, budgets disponibles et choix des moyens utilisés, résultats obtenus, évaluation et éventuellement prospective. Ce plan est simple. Il suit, dans ses grandes lignes, la chronologie des événements. S'il y a un apport d'analyse et de théorie, il se fait lors de la période d'échange entre l'acteur, le professeur et la classe après la présentation du cas.

21 Il n'y a pas de bon cas ou de mauvais cas. Il y a de bons ou de moins bons acteurs dans le cadre pédagogique! Malgré la collaboration entre le professeur et l'acteur avant le cours et la demande de faire une présentation ordonnée, il peut arriver que celle-ci soit brouillonne - désordre chronologique, mélange de plusieurs cas, superficialité du contenu-ou ennuyeuse.

Il est arrivé que le même acteur soit invité l'année suivante avec un cas semblable, par exemple les activités de communication dans le cadre d'une levée de fonds annuelle. L'avantage de cette démarche est que l'acteur assimile mieux le cadre pédagogique et met à profit la période de question de l'année précédente pour monter sa présentation. Le 
désavantage est corollaire. L'acteur prend parfois trop de recul par rapport à son organisation et change de ce fait la valeur pédagogique de son témoignage.

\section{Nouvelles technologies et formation continue}

L'expérience du cours d'études de cas développée au Département d'information et de communication de l'Université Laval a donné naissance à une expérience originale : une étude de cas interactive sur ordinateur.

Une équipe formée d'un informaticien et d'une étudiante à la maîtrise en communication ont mis sur ordinateur un cas complexe - les activités de communication du Carnaval de Québec-. Le logiciel utilisé permet une interactivité. Les étudiants élaborent leurs propres stratégies, moyens et moyens d'évaluation de façon entièrement autonome. Ensuite, en groupe, ces étudiants peuvent mettre en commun les situations qu'ils ou elles ont élaborées. Ce travail de mise en commun repris avec le professeur permet de prendre du recul par rapport au cas et de couvrir une matière beaucoup plus vaste que ce que chacun a découvert. Trop récente, l'expérience n'a été testée que par une dizaine d'étudiants dont les réactions ont été très positives. Ils se sentent à l'aise avec l'appareillage et ont développé leur créativité et leur esprit critique. Pour le moment ce genre d'expérience est encore trop coûteuse en temps de programmation. Mais avec la venue de logiciels plus faciles à utilisés et plus souples l'investissement en temps nécessaire pour ce type de cours-atelier diminuera rapidement. Dès lors le cours pourra facilement se faire à distance en utilisant le réseau internet - par exemple-. Ce pourrait être une formule intéressante à développer. Le contenu du cas comme tel est fait en collaboration avec un " acteur ». La discussion des solutions apportées par l'étudiant peut se faire idéalement en groupe avec un acteur et un professeur. De cette façon, l'apprentissage est de nouveau entre les mains de l'étudiant - ce qui pour moi est la meilleure des solutions - et non entre celle de l'acteur ou du professeur.

\section{Conclusion}

Serait-ce possible, en copiant la démarche des écologistes ou des géologues, de reprendre les études de cas présentées depuis deux ans, une vingtaine, de faire des liens entre elles, de les passer au crible de l'analyse, de les comparer aux théories existantes pour les modifier ou les renouveler? Un bref retour sur la littérature de la méthodologie en sciences sociales et en communication nous en décourage (Bowers, 1984 ; Hsia, 1988). Le nombre de cas est trop petit, le recul trop court (Glaser, Strauss, 1967).

Peut-on envisager de publier les meilleures de ces études de cas en collaboration entre les acteurs et le professeur ? Il y a à cela plusieurs obstacles. Le premier et sûrement le plus important reste celui de la confidentialité. Il y a une grande différence entre venir expliquer une stratégie à une centaine d'étudiants dans un contexte connu et la voir décrite pour un nombre inconnu de lecteurs tout aussi inconnus. Il y a de fortes chances que le texte imprimé soit nettement moins complet, formateur et vivant que l'exposé qui a été fait devant le groupe. Le second obstacle, c'est le facteur actualité. En tenant compte des délais de publication et de la vitesse à laquelle évoluent actuellement les pratiques professionnelles en relations publiques, les cas présentés ne seraient plus actuels et perdraient une grande partie de leur intérêt pédagogique! 


\section{BIBLIOGRAPHIE}

BERG D. N., and K. K. SMITH., Exploring clinical methods for social research, Beverly Hills, Sage publication, editors 1985, 400 pages.

BOWERS J.W. et J.A. COURTRIGHT, Communication Research Methods, Glenview Ill., Scott Foresman, 1984, 367 pages.

DESLAURIERS J.P., Recherche qualitative, Guide pratique, Montréal, McGraw Hill éditeurs, 1991, 142 pages.

GAUTHIER B., sous la direction de, Recherche sociale, de la problématique à la collecte de données, Presses de l'Université du Québec, Sillery, 1993, 584 pages.

GAUTHIER B., « La structure de la preuve », chapitre 6 de Gauthier B., sous la direction de, 1993, Recherche sociale, de la problématique à la collecte de données, Presses de l'Université du Québec, Sillery, 1993, pp. 141 à 174.

GINGRAS P.F., « Sociologie de la connaissance » in Benoit Gauthier, Recherche sociale, de la problématique à la collecte de données, Presses de l'Université du Québec, Sillery, 1993, pp. 19 et 20.

GLASER B.G et A.L. STRAUSS., The discovery of grounded theory, strategies for qualitative research, Aldyne de Gruyter, New-York, 1967, 271 pages.

GOFFMAN E., Les cadres de l'expérience, Les éditions de minuit, Paris, 1991, 573 pages.

HAMON Ph., Du descriptif, Hachette supérieur, Recherches littéraires, Paris, 1993, 247 pages.

HSIA H.J., Mass Communications Research Methods : a step by step approach, Hillsdale N.J., Lawrence Erlbaum ass. publ.,1988, 629 pages.

LARAMEE A. et B. VALLEE., La recherche en communication, Éléments de méthodologie, Sillery, Les Presses de l'Université du Québec, 1991, 377 pages.

LOWMAN R.D., 1985, What is clinical method, in Berg D.N., and K.K. Smith, editors, Exploring clinical methods for social research, Sage publication, Beverly Hills, 1985, pp. 173-190.

MASSE P., Méthodes de collectes et d'analyse de données en communication, Sillery, Les Presses de l'Université du Québec, 1992, 253 pages.

WATT J.H. et S. VAN DEN BERG., Research Methods for Communication science, Boston, Allyn and Bacon, 1995, 468 Pages.

\section{NOTES}

1. Traduction libre de:»In médecine the term clinical refer to those aspects of study that are based on actual treatment and observation of patients as distinguish from experimental or laboratory study » (Lowman, 1985).

2. Comme le présente J-P. Deslauners (1991) dans son manuel sur la recherche qualitative, Weber accordait plus d'importance au sens. Il était nécessaire de prendre en considération la signification subjective de la réalité sociale pour comprendre la société comme telle. Les valeurs, 
les objectifs poursuivis par une personne son interprétation des événements, sa façon de comprendre sa société renseignent sur la réalité sociale.

3. Voir Glaser et Strauss (1967) pour une analyse fort intéressante de l'utilisation des études de cas pour bâtir des théories en sociologie.

4. Décrire ou «de scribere » : étymologiquement : écrire d'après un modèle, dans Hamon 1993 page 48 .

5. Dans un cours il y a habituellement de 10 à 11 leçons disponibles.

6. Selon la personne choisie, le cas sera différent : «lorsque ceux qui participent à une activité jouent des rôles différents, leurs points de vue sur ce qui se passe ont de grande chance d'être aussi différents les uns des autres. Ce qui représente un divertissement pour le joueur de golf est un travail pour le caddy « (Goffman $1991:$ p. 16-17)

7. Goffman (1991), «La définition d'une situation est construite selon des principes d'organisation qui structurent les événements - du moins ceux qui ont un caractère social - et notre propre engagement subjectif. Le terme de « cadre- désigne ces éléments de base », p. 19

\section{RÉSUMÉS}

Les cours "études de cas" font partie de presque tous les curriculums universitaires de formation à des pratiques professionnelles. Quels savoirs sont transmis dans ces cours? Comment le professeur doit-il les mettre en perspective? C'est de cette réflexion qu'il sera question dans cet article.

«Case studies » courses are contort in university programs leading to professional degrees. The present paper looks at a certain number of questions : what kind of knowledge is transmitted and how the professor must put the case in perspective?

\section{AUTEUR}

\section{MARIANNE KUGLER}

Depuis juin 1994, Marianne Kugler est professeur-adjointe au Département d'information et de communication de l'Université Laval à Québec. Elle enseigne plus spécialement aux étudiantes et étudiants des profils marketing social et relations publiques. Docteur de l' Université d'Ottawa pour une thèse sur l'utilisation des bases de données en géologie (1974), elle a été, de 1978 à 1994, conseillère en communication scientifique, puis chef de division de l'information au Service des communications de l'Université Laval. 